www.nature.com/jhg

\title{
A Commentary on Diagnostic and follow-up system for X-linked adrenoleukodystrophy: a comprehensive model for 'treatable' genetic diseases
}

\author{
Takeo Kubota \\ Journal of Human Genetics (2011) 56, 101; doi:10.1038/jhg.2010.149; published online 2 December 2010
}

$\mathrm{I}^{\mathrm{n}}$ $\mathrm{n}$ this issue of the Journal of Human Genetics, Shimozawa et al. ${ }^{1}$ describe a comprehensive diagnostic and follow-up system for X-linked adrenoleukodystrophy (X-ALD) in Japan. The authors present a number of mutation types in the causative $A B C D 1$ gene in Japanese patients, and propose that the genetic testing should be used not only for diagnosis in patients with symptoms but also for a presymptomatic diagnosis in at-risk individuals, as an effective therapy, namely hematopoietic stem cell transplantation (HSCT), is available for this disease. The system proposed by the authors, which consists of metabolic and genetic testing, genetic counseling, therapy and a longitudinal follow-up, will be a model for a genetic disease with effective therapy.

$\mathrm{X}$-ALD is a neurodegenerative disorder associated with the accumulation of very long-chain saturated fatty acids (VLCFAs), and it has several clinical forms, including childhood cerebral form, adrenomyeloneuropathy and Addison's disease (adrenal insufficiency). ${ }^{2}$ Patients are usually male, but some carrier females also develop mild neurological manifestations. The diagnosis of X-ALD is made in combination with clinical findings, brain magnetic resonance imaging, plasma concentration of VLCFAs and molecular genetic testing of $A B C D 1 .{ }^{3} \mathrm{X}-\mathrm{ALD}$ is inherited in an X-linked manner: $93 \%$ of the $\mathrm{X}$-ALD cases are caused by the $A B C D 1$ mutation inherited from one parent and the remaining $7 \%$ are caused by a de novo mutation. Corticosteroid replacement therapy for adrenal insufficiency and HSCT are effective. ${ }^{4,5}$

After the discovery of the causative gene $A B C D 1,{ }^{6,7}$ genetic testing was started. ${ }^{2}$ Once a gene mutation is found in the proband, other family members potentially become a carrier or an at-risk person. In a general genetic disease, genetic testing on other family members should be performed carefully. However, in X-ALD, presymptomatic diagnosis in other family members is useful because an effective therapy (that is HSCT) is available and HSCT can prevent the progression of neurological features if it is applied at an early stage. Hence, it is worth checking the mutation as the number of 'treatable genetic diseases' is increasing, such as Fabry disease with a specific enzymatic therapy. Therefore, a comprehensive system for the diagnosis and follow-up can be developed in other genetic diseases, especially for X-linked ones with asymptomatic carriers. In this context, the comprehensive system described in this review article will be a model for 'treatable' genetic diseases.

1 Shimozawa, N., Honda, A., Kajiwara, N., Kozawa, S., Nagase, T., Takemoto, Y. et al. X-linked adrenoleukodystrophy: Diagnostic and follow-up system in Japan. J. Hum. Genet. 56, 106-109 (2011).

2 Moser, H. W., Raymond, G. V. \& Dubey, P. Adrenoleukodystrophy: new approaches to a neurodegenerative disease. JAMA 294, 3131-3134 (2005).

3 Boehm, C. D., Cutting, G. R., Lachtermacher, M. B., Moser, H. W. \& Chong, S. S. Accurate DNAbased diagnostic and carrier testing for X-linked adrenoleukodystrophy. Mol. Genet. Metab. 66, 128-136 (1999).

4 Shapiro, E., Krivit, W., Lockman, L., Jambaque, I., Peters, C., Cowan, M. et al. Long-term effect of bone-marrow transplantation for childhood-onset cerebral X-linked adrenoleukodystrophy. Lancet 356, 713-718 (2000).

5 Peters, C., Charnas, L. R., Tan, Y., Ziegler, R. S., Shapiro, E. G., DeFor, T. et al. Cerebral X-linked adrenoleukodystrophy: the international hematopoietic cell transplantation experience from 1982 to 1999. Blood 104, 881-888 (2004).

6 Mosser, J., Douar, A. M., Sarde, C. O., Kioschis, P., Feil, R., Moser, H. et al. Putative X-linked adrenoleukodystrophy gene shares unexpected homology with $A B C$ transporters. Nature 361, 726-730 (1993).

7 Uchiyama, A., Suzuki, Y., Song, X. Q., Fukao, T., Imamura, A., Tomatsu, S. et al. Identification of a nonsense mutation in ALD protein cDNA from a patient with adrenoleukodystrophy. Biochem. Biophys. Res. Commun. 198, 632-636 (1994). 